\title{
A retrospective, matched cohort study of potential drug-drug interaction prevalence and opioid utilization in a diabetic peripheral neuropathy population initiated on pregabalin or duloxetine
}

Jeffrey J Ellis ${ }^{1 *}$, Alesia B Sadosky², Laura L Ten Eyck ${ }^{3}$, Pallavi Mudumby ${ }^{1}$, Joseph C Cappelleri², Lilian Ndehi', Brandon T Suehs ${ }^{1}$ and Bruce Parsons ${ }^{2}$

\begin{abstract}
Background: Anticipating and controlling drug-drug interactions (DDIs) in older patients with painful diabetic peripheral neuropaty (pDPN) presents a significant challenge to providers. The purpose of this study was to examine the impact of newly initiated pregabalin or duloxetine treatment on Medicare Advantage Prescription Drug (MAPD) plan pDPN patients' encounters with potential drug-drug interactions, the healthcare cost and utilization consequences of those interactions, and opioid utilization.
\end{abstract}

Methods: Study subjects required a pregabalin or duloxetine pharmacy claim between 07/01/2008-06/30/2012 (index event), $\geq 1$ inpatient or $\geq 2$ outpatient medical claims with pDPN diagnosis between 01/01/2008-12/31/ 2012 , and $\geq 12$ months pre- and $\geq 6$ post-index enrollment. Propensity score matching was used to balance the pregabalin and duloxetine cohorts on pre-index demographics and comorbidities. Potential DDIs were defined by Micromedex 2.0 and identified by prescription claims. Six-month post-index healthcare utilization (HCU) and costs were calculated using pharmacy and medical claims.

Results: No significant differences in pre-index demographics or comorbidities were found between pregabalin subjects $(n=446)$ and duloxetine subjects $(n=446)$. Potential DDI prevalence was significantly greater $(p<0.0001)$ among duoxetine subjects (56.7\%) than among pregabalin subjects (2.9\%). There were no significant differences in HCU or costs between pregablin subjects with and without a potential DDI. By contrast, duloxetine subjects with a potential DDI had higher mean all-cause costs $(\$ 13,908 \mathrm{vs.} \$ 9,830 ; p=0.001)$, more subjects with $\geq 1$ inpatient visits (35.6\% vs $25.4 \% ; p=0.02$ ), and more subjects with $\geq 1$ emergency room visits (32.8\% vs. $20.7 \% ; p=0.005$ ) in comparison to duloxetine subjects without a potential DDI. There was a trend toward a difference between pregabalin and duloxetine subjects in their respective pre-versus-post differences in milligrams (mg) of morphine equivalents/ 30 days used (60.2 $\mathrm{mg}$ and $176.9 \mathrm{mg}$, respectively; $\mathrm{p}=0.058$ ).

Conclusion: The significantly higher prevalence of potential DDIs and potential cost impact found in pDPN duloxetine users, relative to pregabalin users, underscore the importance of considering DDls when selecting a treatment.

Keywords: Healthcare costs, Healthcare utilization, Medicare, Morphine equivalents, Drug-drug interaction, Pregabalin, Duloxetine

\footnotetext{
* Correspondence: jellis21@humana.com

${ }^{1}$ Comprehensive Health Insights Inc., 325 West Main Street WFP6W,

Louisville, KY 40202, USA

Full list of author information is available at the end of the article
}

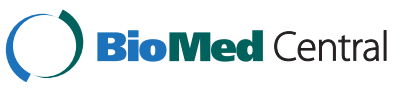

(C) 2015 Ellis et al.; licensee BioMed Central. This is an Open Access article distributed under the terms of the Creative Commons Attribution License (http://creativecommons.org/licenses/by/4.0), which permits unrestricted use, distribution, and reproduction in any medium, provided the original work is properly credited. The Creative Commons Public Domain Dedication waiver (http://creativecommons.org/publicdomain/zero/1.0/) applies to the data made available in this article, unless otherwise stated. 


\section{Background}

Challenging to patients, as well as providers, diabetes has the potential to cause a specific group of nerve disorders categorized as autonomic, peripheral proximal, focal, and peripheral neuropathies [1,2]. A common complication of diabetes, painful diabetic peripheral neuropathy (pDPN) affects approximately 1 in 4 people with this disease. Reduced glycemic control, advanced age of the patient, and the length of time the patient has diabetes are all documented risk factors for developing pDPN [3-6]. Diagnosis of the condition involves documentation of the symptoms of peripheral nerve dysfunction after exclusion of other potential causes of pain. The development of pDPN has been linked to signaling impairment in the central nervous system, although the process driving this change remains inadequately understood $[7,8]$.

While the advancement of pDPN has been shown to be decreased through glycemic control [5], a variety of treatment options are available for pDPN; however, only a limited number have been proven effective in appropriate clinical trial settings $[9,10]$. There are a number of drug classes recommended and available including anticonvulsants, tricyclic antidepressants, and serotonin-norepinephrine reuptake inhibitors (SNRIs) [9,11]. Guidelines are available for treatment of pDPN; however, these can differ, and the recommended medications may generate adverse effects in conjunction with diabetic treatments $[11,12]$. Only three drugs, pregabalin, duloxetine, as well as the recently approved tapentadol, have been approved by the Food and Drug Administration for treatment of pDPN [13-20]. Evidence has been put forth for the use of opioids such as oxycodone and tramadol in treatment of pDPN through randomized controlled trials, although there is no longterm evidence of their impact on the disease or on quality of life of patients [9,11,21-24]. Stemming from the variable course of pDPN, the risk of opioid addiction in this group is increased, and a number of side effects are associated with opioids including hypogonadism and lowered immunity [25-27]. Consequences of opioid overdosing, especially in older patients, can be severe and clinical decision-making must balance the risk of such adverse effects by achieving pain control at the lowest possible combined dose(s) of opioid [28]. Previous retrospective studies across various patient populations and utilizing disparate definitions of opioid utilization have incongruent findings regarding the opioid-sparing effects of pregabalin [29-32] and duloxetine $[29,30,33]$. While clarity on the opioid-sparing effect of pregabalin and duloxetine should continue to be sought, it must be done so in light of introducing potential drug-drug interactions (DDIs) through use of either agent.
As the occurrence of pDPN increases with the degree of hyperglycemia and age of the patient, the total number of prescriptions a patient may be taking has the potential to rise dramatically due to the need to manage the effects of advancing diabetes and comorbidities related to the disease. As such, anticipating and controlling DDIs in patients with pDPN presents a significant challenge to providers, particularly in an older population. The consequences of these drug interactions have the potential to impact the quality of life of the patient, as well as increase the cost of the disease [9,34].

While some studies have examined aspects of treatment outcomes such as healthcare utilization and economic comparisons between pregabalin and duloxetine (where the costs were found to be comparable between the two drugs), few studies have documented the costs incurred from DDIs related to treatment of patients with pDPN [29,34-36]. Recently, Johnston et al. [34] examined the frequency and financial impact of DDIs and drug-condition interactions (DCIs) in predominantly commercially-insured pDPN patients prescribed pregabalin or duloxetine and found patients taking duloxetine had a substantially higher potential for DDIs and DCIs than patients taking pregabalin. Furthermore, the costs associated with potential DDIs and DCIs in patients taking duloxetine were found to be significantly increased.

The present study continues and builds on previous research by examining the impact of newly initiated pregabalin or duloxetine treatment on Medicare Advantage Prescription Drug plan (MAPD) pDPN members' encounters with potential drug-drug interactions, the healthcare cost and utilization consequences of those interactions, and opioid utilization.

\section{Methods}

\section{Study design and subject selection}

This study is a retrospective, matched cohort study using medical and pharmacy claims collected from a large MAPD health plan. Medical and pharmacy claims data were extracted for the period of time between January 1 , 2007 and December 31, 2012, and these data were used to identify potential subjects, measure patient characteristics, and examine study outcomes. MAPD members diagnosed with DPN were identified as potential subjects for the analysis based on International Classification of Disease, 9th revision, Clinical Modification (ICD-9-CM) diagnosis data reported on medical claims.

Members receiving pregabalin $\left(\right.$ Lyrica $\left.^{\circ}\right)$ or duloxetine (Cymbalta ${ }^{\circ}$ ) between January 1, 2008 and June 30, 2012 were identified based on pharmacy claims, and the first observed prescription claim during this period was assigned as the study index date. Tapentadol was not included as a comparator as the study period did not allow for sufficient sample size to analyze members prescribed 
that agent. Identified members were further required to have 12 months of continuous pre-index enrollment during which no prescription claim for duloxetine or pregabalin was observed.

In order to enhance the likelihood that the medication of interest was being used to treat $\mathrm{pDPN}$, it was further required that a diagnosis of DPN be observed within 90 days of the index date. Subjects meeting any of the following criteria were excluded from the study: Age $<18$ or $>89$ years; continuous enrollment $<6$ months postindex; diagnosis of fibromyalgia (FM) at any time after the index date; diagnosis and/or procedure code indicative of transplant surgery, cancer, or pregnancy during study period; long-term care facility residence of $\geq 90$ days during the study period; or, one or more prescription claims for the non-index comparator drug (eg, if index drug is duloxetine, then non-index comparator drug is pregabalin) 12 months prior to or on the index date. The research protocol was reviewed and approved by Shulman Associates IRB, an independent Institutional Review Board, prior to study initiation. A waiver of informed consent and a waiver of authorization to use protected health information (PHI) were granted.

\section{Baseline measures}

The subject's assignment to either the pregabalin cohort or the duloxetine cohort was determined by the medication filled on the subject's index date. Age, gender, race/ ethnicity, geographic region of residence, low income subsidy (LIS) status, and Medicare dual eligibility (DE) status were measured based on member enrollment data. The Deyo implementation of the Charlson comorbidity index score was calculated based on medical claims adjudicated (insurer payment determined) during the pre-index observation period $[37,38]$. In addition to the Charlson score, specific pain-related medical conditions of interest were identified based on ICD-9-CM codes reported on medical claims during the pre-index period.

Pre-index and post-index medication utilization were measured based on pharmacy claims adjudicated during their respective observation periods. Pain-related medication use patterns were measured for the following medication classes: opioids, non-opioid analgesics, antidepressants, anticonvulsants, anesthetics, muscle relaxants, and anxiolytics. Individual subjects were identified as medication users if they had one or more prescription claims for the specific medication during the observation period. Opioid use was further examined and categorized as long-acting, short-acting low potency, and short-acting high potency [39]. In addition, intensity of opioid use was determined through conversion to morphine equivalents (MEq) based on established conversion factors [39]. The opioid dose strength and quantity of fill for each adjudicated opioid prescription claim was converted to $\mathrm{MEq}$, summed for the observation period, and then normalized to an average MEq per 30 days.

\section{Study outcomes}

\section{Drug-drug interaction status}

Medications with high potential for drug-drug interactions (DDI) with duloxetine or pregabalin (Table 1) were identified based on a drug interaction report generated from Micromedex 2.0 (Truven Health Analytics Inc., Greenwood Village, CO). Medications with an interaction classified as contraindicated, major, and moderate were included as potentially interacting drugs of interest. Medications with an interaction classified as minor or unknown in the Micromedex 2.0 system were not included as potentially interacting medications. Prescription claims were used to identify subjects receiving medication(s) that potentially interact with the index medication.

Subjects were flagged with a DDI if they met either of the following criteria: 1) a prescription claim for an interacting medication during the pre-index period and a corresponding days' supply indicative of coverage that overlapped the index date; or, 2) an adjudicated prescription claim for an interacting medication filled within 30 days after the index date.

\section{Healthcare resource utilization and costs}

All-cause medical service utilization and costs were measured using medical claims data. Medical claim place of service was used to assign inpatient, emergency room, and outpatient utilization and costs. Physical therapy (PT) utilization was identified using medical claims where the Current Procedural Terminology (CPT) code billed indicated a physical therapy service was performed. Total pharmacy costs were defined as the sum of costs (plan-paid and member-paid) associated with adjudicated pharmacy claims. Total all-cause healthcare costs were defined as the sum of the respective total medical cost and total pharmacy cost components. All cost calculations included both member and plan paid components, and were adjusted to 2012 United States (US) dollars based on the medical care component of the Consumer Price Index. Post-index opioid utilization was determined both categorically and as MEq per 30 days, as previously described for the pre-index period.

\section{Analysis}

Propensity score (PS) matching was utilized to address potential bias in this non-randomized, observational study $[40,41]$. In the PS matching, all baseline demographic characteristics and medical conditions of interest were included. The PS matching process, matching a 
Table 1 Pregabalin and duloxetine drug-drug interactions

\begin{tabular}{|c|c|c|}
\hline Severity & Brief description of potential harm due to DDI & Interacting drug \\
\hline \multicolumn{3}{|l|}{ Pregabalin } \\
\hline Major & Reduced pregabalin effectiveness & naproxen, ketorolac \\
\hline \multicolumn{3}{|l|}{ Duloxetine } \\
\hline \multirow[t]{4}{*}{ Contraindicated } & CNS toxicity or serotonin syndrome & isocarboxazid, linezolid, procarbazine, rasagiline, selegiline, tranylcypromine \\
\hline & $\begin{array}{l}\text { Increased serum concentrations of interacting drug } \\
\text { and risk of cardiac arrhythmia }\end{array}$ & thioridazine \\
\hline & $\begin{array}{l}\text { Increased risk of extrapyramidal reactions or } \\
\text { neuroleptic malignant syndrome }\end{array}$ & metoclopramide \\
\hline & $\begin{array}{l}\text { Increased risk of serotonin syndrome or neuroleptic } \\
\text { malignant syndrome-like reactions }\end{array}$ & methylene blue \\
\hline \multirow[t]{5}{*}{ Major } & $\begin{array}{l}\text { Increased interacting drug plasma level and } \\
\text { Increased risk of QT prolongation }\end{array}$ & clozapine \\
\hline & Increased risk of bleeding & antiplatelet agents, escitalopram \\
\hline & Increased risk of serotonin syndrome & $\begin{array}{l}\text { almotriptan, citalopram, cyclobenzaprine, desvenlafaxine, dextromethorphan, } \\
\text { eletriptan, fluoxetine, fluvoxamine, frovatriptan, hydroxytryptophan, lithium, } \\
\text { lorcaserin, methadone, milnacipran, naratriptan, paroxetine, rizatriptan, sertraline, } \\
\text { sumatriptan, tapentadol, tramadol, trazodone, tryptophan, venlafaxine, zolmitriptan }\end{array}$ \\
\hline & $\begin{array}{l}\text { Increased risk of serotonin syndrome or neuroleptic } \\
\text { malignant syndrome-like reactions }\end{array}$ & vilazodone \\
\hline & $\begin{array}{l}\text { Increased serum concentrations of interacting drugs } \\
\text { and an Increased risk of cardiotoxicity }\end{array}$ & class $1 \mathrm{C}$ antiarrhythmic agents \\
\hline \multirow[t]{4}{*}{ Moderate } & $\begin{array}{l}\text { decreased plasma concentrations of the active } \\
\text { metabolites of interacting drug }\end{array}$ & tamoxifen \\
\hline & $\begin{array}{l}\text { Increased duloxetine serum concentrations and risk } \\
\text { of adverse effects }\end{array}$ & ciprofloxacin, clobazam, enoxacin, mirabegron, quinidine \\
\hline & $\begin{array}{l}\text { Increased exposere to interacting drug and } \\
\text { potential toxicity }\end{array}$ & phenothiazines, tamsulosin, tricyclic antidepressants \\
\hline & Increased risk of bleeding & $\begin{array}{l}\text { acenocoumarol, dabigatran, dalteparin, danaparoid, desirudin, enoxaparin, } \\
\text { fondaparinux, nonsteroidal anti-inflammatory agents, phenindione, } \\
\text { phenprocoumon, tinzaparin, warfarin }\end{array}$ \\
\hline
\end{tabular}

Source: Micromedex 2.0 (Truven Health Analytics Inc., Greenwood Village, CO), accessed May 31, 2013. Drugs with primary use in the hospital setting (e.g., lepirudin) were excluded.

duloxetine member to a pregabalin member, was based on the nearest neighbor approach, without replacement, using a caliper width of 0.005 .

Diagnostic evaluation was considered to examine balance in pre-index covariates (from the propensity score model) between matched treatment groups. In order to account for the non-normal data distribution, continuous pre-index measures were compared between-groups using non-parametric Wilcoxon rank-sum tests. Chisquare tests were used to compare categorical pre-index data. Wilcoxon rank-sum test and chi-square tests were also performed for post-index outcomes [42].

Multivariable statistical models were applied to examine the relationship between DDI status and healthcare costs [43]. Specifically, a generalized linear mixed model (GLMM) with a gamma distribution and log link was fitted to model total healthcare costs. Several variables were entered into the model. They included index drug, DDI status, demographic characteristics, baseline medical conditions, and pre-index medication utilization. An "index drug*DDI status" covariate interaction term was also included in the model. Means and confidence intervals were derived from the models and then retransformed into dollar values through exponentiation of the adjusted least square (LS) means estimates. All comparisons of total all-cause healthcare costs between and within cohorts were assessed using Wald chi-square tests.

Differences in all-cause total healthcare costs were compared between the cohorts (i.e., by pregabalin/duloxetine and DDI status) via Difference-in-Differences (DID) analyses, using the model-based LS Means estimates and corresponding standard errors:

DID = mean of values in (DDI present minus DDI absent in the pregabalin cohort) minus mean of values in (DDI present minus DDI absent in the duloxetine cohort).

T-tests were performed to formally test the DID values for statistical significance.

Differences in pre-index and post-index opioid utilization and MEq were compared between pregabalin and duloxetine cohorts via DID analysis. The t-statistic 
for the opioid utilization DID analysis was derived from a GLMM with a logit link and binary distribution, controlling for baseline demographics, Deyo-Charlson comorbidity index, and pre- and post-index non-opioid pain medication utilization. The t-statistic for the MEq DID analysis was derived from a GLMM with an identity link and a Gaussian distribution, controlling for baseline demographics, Deyo-Charlson comorbidity index, and preand post-index non-opioid pain medication utilization. The MEq DID analysis covered opioid prescription claims from 6-months pre-index to 6-months post-index.

All data analyses were conducted using SAS version 9.3/SAS Enterprise Guide 5.1. The a priori alpha level for all inferential analyses was set at 0.05; all statistical tests were two-tailed.

\section{Results}

Patient characteristics

A total of 1,320 subjects met the study inclusion and exclusion criteria (pregabalin $n=570$, duloxetine $n=750$ ). After conducting the propensity score matching procedure, the analytic cohort consisted of 446 matched pairs (892 total study subjects). The lack of statistically significant differences between the matched cohorts on baseline demographics (see Table 2) or pre-index comorbidity measures (see Table 3) provides evidence to support that the matching process was successful. The predominantly older composition of the study cohorts was confirmed as both the pregabalin and duloxetine groups exhibited a mean age of 66.7 years. The percentage of subjects with a potential DDI was greater in the duloxetine group compared to the pregabalin group $(56.7 \%, \mathrm{n}=253$ vs. $2.9 \%, \mathrm{n}=13, \mathrm{p}<0.001)$. There were 17 potential DDIs involving pregabalin, all of which were of "Major" severity. There were 649 potential DDIs involving duloxetine, including 11 "Contraindicated" interactions and 453 "Major" interactions (Table 4).

\section{Healthcare resource utilization}

There were no significant differences in any form of healthcare utilization between pregabalin subjects with and without a potential DDI (see Table 5). Compared to duloxetine subjects without a potential DDI, significantly greater percentages of duloxetine subjects with a potential DDI had at least one inpatient hospitalization and at least one emergency room (ER) visit. Similarly, duloxetine subjects with a potential DDI had significantly higher mean numbers of visits per member for all-cause inpatient hospitalizations, ER visits, and outpatient (non-physical therapy) visits compared to those without a potential DDI.

\section{Healthcare costs}

There were no significant differences in all-cause healthcare costs, or component costs (eg, pharmacy), between
Table 2 Patient characteristics

\begin{tabular}{|c|c|c|c|c|c|}
\hline & \multirow{2}{*}{\multicolumn{2}{|c|}{$\begin{array}{l}\text { Pregabalin } \\
N=446\end{array}$}} & \multirow{2}{*}{\multicolumn{2}{|c|}{$\begin{array}{l}\text { Duloxetine } \\
N=446\end{array}$}} & \multirow[b]{3}{*}{$P$ value* } \\
\hline & & & & & \\
\hline & $n$ & $\%$ & $n$ & $\%$ & \\
\hline \multicolumn{5}{|l|}{ Age, years (categorical) } & \multirow[t]{8}{*}{0.1304} \\
\hline $18-29$ & 0 & 0.0 & 0 & 0.0 & \\
\hline $30-39$ & 7 & 1.6 & 1 & 0.2 & \\
\hline $40-49$ & 27 & 6.1 & 26 & 5.8 & \\
\hline $50-59$ & 73 & 16.4 & 86 & 19.3 & \\
\hline $60-69$ & 144 & 32.3 & 159 & 35.7 & \\
\hline $70-79$ & 150 & 33.6 & 126 & 28.3 & \\
\hline $80-89$ & 45 & 10.1 & 48 & 10.8 & \\
\hline \multicolumn{5}{|l|}{ Gender } & \multirow[t]{3}{*}{0.5854} \\
\hline Male & 185 & 41.5 & 177 & 39.7 & \\
\hline Female & 261 & 58.5 & 269 & 60.3 & \\
\hline \multicolumn{5}{|l|}{ Race/Ethnicity } & \multirow[t]{5}{*}{0.7265} \\
\hline White & 363 & 81.4 & 353 & 79.1 & \\
\hline Black & 58 & 13.0 & 69 & 15.5 & \\
\hline Hispanic & 13 & 2.9 & 14 & 3.1 & \\
\hline Other & 12 & 2.7 & 10 & 2.2 & \\
\hline \multicolumn{5}{|l|}{ Geographic Region } & \multirow[t]{5}{*}{0.6759} \\
\hline Northeast & 5 & 1.1 & 5 & 1.1 & \\
\hline Midwest & 78 & 17.5 & 78 & 17.5 & \\
\hline South & 338 & 75.5 & 333 & 74.7 & \\
\hline West & 25 & 5.6 & 30 & 6.7 & \\
\hline Low Income Status & 87 & 19.5 & 92 & 20.6 & 0.6814 \\
\hline \multirow[t]{2}{*}{ Dual Eligibility Status } & 52 & 11.7 & 56 & 12.6 & 0.6759 \\
\hline & Mean & SD & Mean & SD & \\
\hline Age, years (continuous) & 66.7 & 10.7 & 66.7 & 10.4 & 0.5924 \\
\hline Propensity Score & 0.47 & 0.14 & 0.47 & 0.14 & 0.9931 \\
\hline
\end{tabular}

*All $\mathrm{P}$ values for categorical variables calculated using chi -square tests. All $P$ values for continuous variables calculated using Wilcoxon rank-sum tests. SD, Standard Deviation.

pregabalin subjects with and without a potential DDI (see Table 6). Conversely, duloxetine subjects with a potential DDI exhibited significantly greater costs in comparison to duloxetine subjects without a potential DDI. Among duloxetine subjects, all-cause total healthcare costs were significantly higher for those with a potential DDI than those without (means: $\$ 13,908$ vs. $\$ 9,830$; $p=$ 0.001). The component healthcare costs of total medical, inpatient-related, ER-related, and total pharmacy costs were also significantly higher in duloxetine subjects with a potential DDI compared to those without.

\section{Model-adjusted healthcare costs}

No significant differences were found in model-adjusted mean all-cause healthcare costs for subjects with a potential DDI versus those subjects without a potential 
Table 3 Pre-index comorbid conditions

\begin{tabular}{|c|c|c|c|c|c|}
\hline & \multirow{2}{*}{\multicolumn{2}{|c|}{$\frac{\text { Pregabalin }}{N=446}$}} & \multirow{2}{*}{\multicolumn{2}{|c|}{$\frac{\text { Duloxetine }}{N=446}$}} & \multirow[b]{3}{*}{$P$ value* } \\
\hline & & & & & \\
\hline & $n$ & $\%$ & $n$ & $\%$ & \\
\hline Abdominal pain/cramping & 106 & 23.8 & 107 & 24.0 & 0.9374 \\
\hline Anxiety disorder & 57 & 12.8 & 57 & 12.8 & 1 \\
\hline Arthritis & 186 & 41.7 & 198 & 44.4 & 0.4171 \\
\hline $\begin{array}{l}\text { Back pain with neuropathic } \\
\text { involvement }\end{array}$ & 123 & 27.6 & 126 & 28.3 & 0.8228 \\
\hline $\begin{array}{l}\text { Causalgia and other painful } \\
\text { neuropathies }\end{array}$ & 109 & 24.4 & 112 & 25.1 & 0.8160 \\
\hline Depression & 77 & 17.3 & 71 & 15.9 & 0.5892 \\
\hline Diabetes & 388 & 87.0 & 397 & 89.0 & 0.3537 \\
\hline Epilepsy & 9 & 2.0 & 10 & 2.2 & 0.8166 \\
\hline Fatigue & 133 & 29.8 & 136 & 30.5 & 0.8268 \\
\hline Irritable bowel syndrome & 6 & 1.3 & 6 & 1.3 & 1 \\
\hline Migraine headache & 9 & 2.0 & 10 & 2.2 & 0.8166 \\
\hline Muscle weakness & 50 & 11.2 & 49 & 11.0 & 0.9151 \\
\hline $\begin{array}{l}\text { Neck pain with neuropathic } \\
\text { involvement }\end{array}$ & 36 & 8.1 & 42 & 9.4 & 0.4770 \\
\hline Other back pain & 165 & 37.0 & 168 & 37.7 & 0.8355 \\
\hline Other mental disorders & 1 & 0.2 & 1 & 0.2 & $* *$ \\
\hline Other musculoskeletal pain & 333 & 74.7 & 338 & 75.8 & 0.6982 \\
\hline Other neck pain (spinal) & 57 & 12.8 & 63 & 14.1 & 0.5560 \\
\hline Sleep disorders & 111 & 24.9 & 114 & 25.6 & 0.8171 \\
\hline Substance abuse or dependence & 57 & 12.8 & 59 & 13.2 & 0.8422 \\
\hline Tension headache & 5 & 1.1 & 4 & 0.9 & $* *$ \\
\hline Thinking/memory loss & 12 & 2.7 & 8 & 1.8 & 0.3657 \\
\hline \multirow[t]{2}{*}{ Trigeminal nerve disorders } & 2 & 0.4 & 0 & 0.0 & ** \\
\hline & Mean & SD & Mean & SD & \\
\hline $\begin{array}{l}\text { Deyo-Charlson Comorbidity } \\
\text { Index score }\end{array}$ & 2.6 & 2.2 & 2.7 & 2.2 & 0.3058 \\
\hline
\end{tabular}

*All $P$ values for categorical variables calculated using chi-square tests. All $P$ values for continuous variables calculated using Wilcoxon rank-sum tests. **Expected Counts $<5$. Chi-square test may not be appropriate.

SD, Standard Deviation.

DDI in either the pregabalin cohort $(\$ 33,942$ vs. $\$ 31,878$, respectively; $\mathrm{p}=0.8159)$ or the duloxetine cohort $(\$ 39,445$ vs. $\$ 36,424$, respectively; $\mathrm{p}=0.4208)$ (see Table 7$)$. In accordance with those findings, the DID analysis found no significant difference in all-cause healthcare costs associated with the presence of a potential DDI attributable to the index medication $(\mathrm{p}=0.8950)$.

\section{Opioid utilization and morphine equivalents}

The DID analyses for the associations of index medication with opioid utilization and morphine equivalents utilization are described in Tables 8 and 9, respectively. There was a trend toward a significant difference between pregabalin and duloxetine subjects in their respective pre-versus-post differences in mean morphine
Table 4 Observed pregabalin and duloxetine drug-drug interactions by severity and interacting drug

\begin{tabular}{|c|c|c|}
\hline Severity* & Interacting drug & $\begin{array}{l}\text { Number of } \\
\text { interactions }\end{array}$ \\
\hline \multicolumn{3}{|l|}{ Pregabalin } \\
\hline \multirow[t]{3}{*}{ Major } & naproxen & 17 \\
\hline & Total Major for pregabalin & 17 \\
\hline & Grand total for pregabalin & 17 \\
\hline \multicolumn{3}{|l|}{ Duloxetine } \\
\hline \multirow[t]{2}{*}{ Contraindicated } & metoclopramide & 11 \\
\hline & Total Contraindicated for duloxetine & 11 \\
\hline Major & tramadol & 35 \\
\hline Major & trazodone & 31 \\
\hline Major & cyclobenzaprine & 31 \\
\hline Major & meloxicam & 29 \\
\hline Major & citalopram & 25 \\
\hline Major & clopidogrel & 91 \\
\hline Major & methadone & 21 \\
\hline Major & fluoxetine & 25 \\
\hline Major & ibuprofen & 22 \\
\hline Major & escitalopram & 11 \\
\hline Major & sertraline & 32 \\
\hline Major & diclofenac & 18 \\
\hline Major & paroxetine & 17 \\
\hline Major & celecoxib & 9 \\
\hline Major & naproxen & 9 \\
\hline Major & venlafaxine & 12 \\
\hline Major & nabumetone & 4 \\
\hline Major & etodolac & 3 \\
\hline Major & piroxicam & 3 \\
\hline Major & lithium & 4 \\
\hline Major & indomethacin & 3 \\
\hline Major & cilostazol & 10 \\
\hline Major & sulindac & 6 \\
\hline \multirow[t]{2}{*}{ Major } & propafenone & 2 \\
\hline & Total Major for duloxetine & 453 \\
\hline Moderate & amitriptyline & 40 \\
\hline Moderate & warfarin & 59 \\
\hline Moderate & promethazine & 15 \\
\hline Moderate & ciprofloxacin & 14 \\
\hline Moderate & tamsulosin & 25 \\
\hline Moderate & doxepin & 8 \\
\hline Moderate & nortriptyline & 6 \\
\hline Moderate & prochlorperazine & 3 \\
\hline Moderate & enoxaparin & 9 \\
\hline Moderate & imipramine & 1 \\
\hline Moderate & dabigatran & 1 \\
\hline
\end{tabular}


Table 4 Observed pregabalin and duloxetine drug-drug interactions by severity and interacting drug (Continued)

Moderate

butalbital

4

Total Moderate for duloxetine

Grand total for duloxetine

649

*Severity category according to Micromedex 2.0 (Truven Health Analytics Inc. Greenwood Village, CO), as of May 31, 2013.

equivalents per 30 days $[60.2$ milligramsn $(\mathrm{mg})$ and $176.9 \mathrm{mg}$, respectively; $\mathrm{p}=0.058$ ]. The DID analysis of pre-index and post-index opioid utilization, as defined by one or more claims for long-acting, short-acting less potent, and short-acting more potent opioids, found no significant difference associated with the index medication.

\section{Discussion}

The present study adds to the limited understanding of potential DDI prevalence, as well as the healthcare cost and utilization consequences of those interactions, in a predominantly older MAPD patient population newly initiated on pregabalin or duloxetine for the treatment of pDPN.

This present study found that pDPN subjects initiating treatment with pregabalin were significantly less likely to encounter potential DDIs than patients initiating duloxetine. The magnitude of DDI prevalence for duloxetine patients observed (56.7\%) is likely driven by factors inherent to the medication itself and by the population in which it was studied. Duloxetine undergoes hepatic metabolism by cytochrome P-450 (CYP450) isoenzymes 2D6 and 1A2. Further, it inhibits isoenzymes 2D6 and $1 \mathrm{~A} 2$ and is highly plasma protein bound resulting in many potential DDIs with other medications [44]. Alternatively, pregabalin has minimal pharmacokinetic or pharmacodynamic interactions with other medications as signified in this study by the low prevalence of potential DDIs found (2.9\%) [45]. The comorbidity burden and associated medication use of the matched pDPN pregabalin and duloxetine cohorts align with previous studies of pDPN patients [29,34-36] and it has been shown that pain treatment itself is an independent risk factor for exposure to potential DDIs $[46,47]$.

A recent study by Johnston et al. found the frequency of potential contraindicated, major, and moderate DDIs in DPN patients newly initiated on duloxetine to be $3.9 \%, 36.9 \%$, and $33.8 \%$, respectively [34]. Conversely, no DPN patients newly initiated on pregabalin encountered a potential DDI of any severity. Methodological variations between the aforementioned study [34] and present study may explain the differences in reported potential DDI prevalence, with the most noteworthy variation being the insurance plan case-mix amongst the study populations. The former study [34] was conducted in a younger, predominantly commercially-insured population while the present study was conducted in a Medicare population with a mean age of 66.7 years for the matched cohorts. This observation highlights the age-related vulnerability to drug interactions experienced by older patients $[48,49]$.

Table 5 6-month post-index all-cause healthcare utilization stratified by drug-drug interaction status

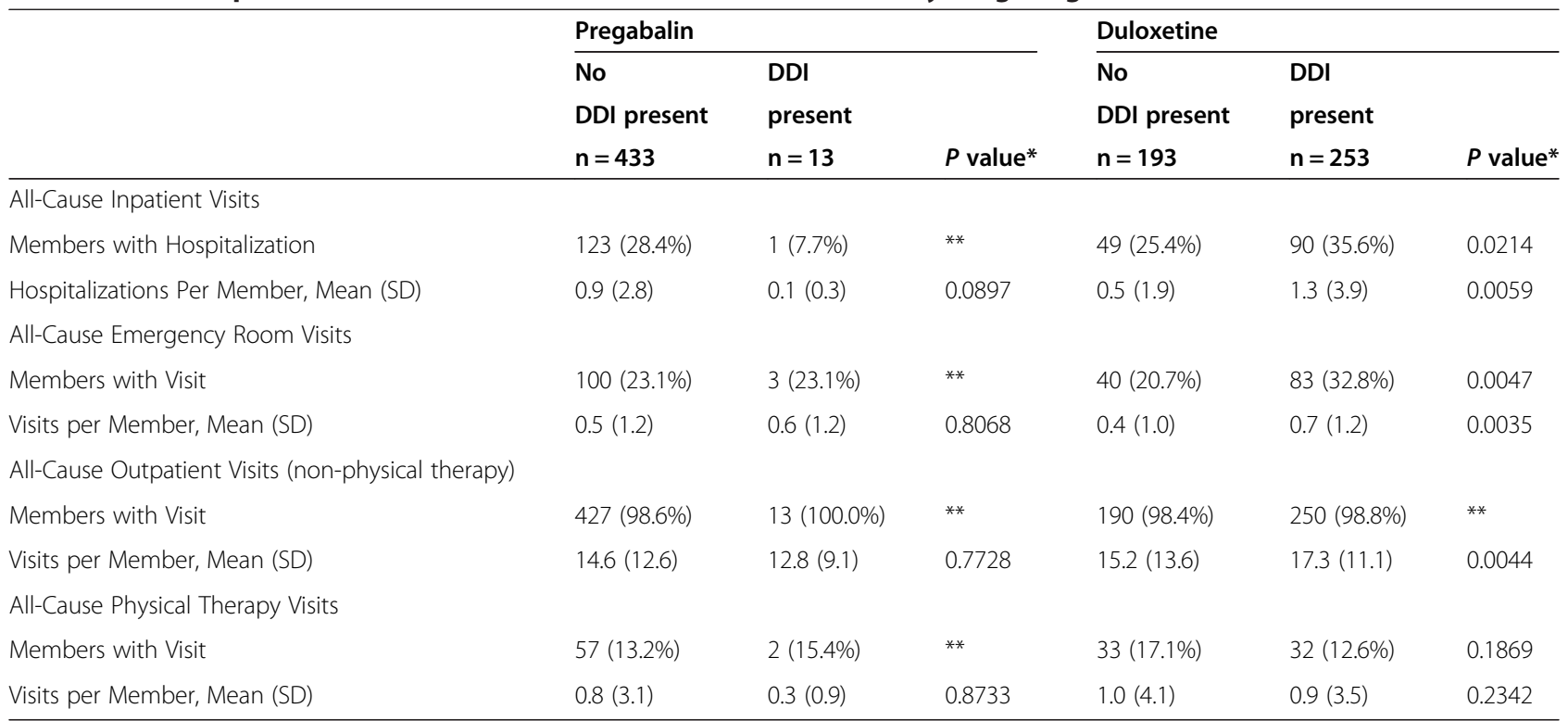

*All P values for categorical variables calculated using Chi Square tests. All P values for continuous variables calculated using Wilcoxon Rank-Sum tests. **Expected Counts $<5$. Chi-square test may not be appropriate. DDI, Drug-Drug Interaction.

SD, Standard Deviation. 
Table 6 6-month post-index all-cause healthcare costs and component costs stratified by drug-drug interaction status

\begin{tabular}{|c|c|c|c|c|c|c|}
\hline & \multicolumn{3}{|l|}{ Pregabalin } & \multicolumn{3}{|l|}{ Duloxetine } \\
\hline & $\begin{array}{l}\text { No DDI present } \\
n=433\end{array}$ & $\begin{array}{l}\text { DDI present } \\
n=13\end{array}$ & $P$ value* & $\begin{array}{l}\text { No DDI present } \\
n=193\end{array}$ & $\begin{array}{l}\text { DDI present } \\
n=253\end{array}$ & $P$ value* \\
\hline All-Cause Total Healthcare Costs, Mean (SD) & $11,085(16,820)$ & $8,470(13,491)$ & 0.2156 & $9,830(14,351)$ & $13,908(20,254)$ & 0.001 \\
\hline All-Cause Total Medical Costs, Mean (SD) & $8,339(16,20)$ & $6,115(13,461)$ & 0.1835 & $7,550(14,081)$ & $10,311(16,609)$ & 0.0278 \\
\hline Outpatient-Related (non-physical therapy), Mean (SD) & $2,819(5,639)$ & $1,944(2,366)$ & 0.7170 & $2,711(4,392)$ & $2,517(3,999)$ & 0.8396 \\
\hline Physical Therapy-Related, Mean (SD) & $93(385)$ & $17.7(64.0)$ & 0.5499 & $98(363)$ & $103(516)$ & 0.2824 \\
\hline Inpatient-Related, Mean (SD) & $4,082(12,371)$ & $3,722.5(13,421.7)$ & 0.1458 & $3,782(12,713)$ & $5,873(13,965)$ & 0.0201 \\
\hline ER-Related, Mean (SD) & $248(700)$ & $217.6(429.6)$ & 0.9152 & $224(675)$ & $386(900)$ & 0.0061 \\
\hline All-Cause Total Pharmacy Costs, Mean (SD) & $2,746(3,213)$ & $2,355.9(2,686.5)$ & 0.3120 & $2,280(2,396)$ & $3,597(11,125)$ & $<0.0001$ \\
\hline
\end{tabular}

Costs in US Dallars (\$).

${ }^{*}$ All $\mathrm{P}$ values for categorical variables calculated using chi-square tests. All P values for continuous variables calculated using Wilcoxon rank-sum tests.

DDI, Drug-Drug Interaction; SD, Standard Deviation.

Previous research reported no statistically significant differences in post-initiation all-cause healthcare costs for pDPN patients newly initiated on pregabalin or duloxetine $[29,36]$. The present study endeavored to determine the impact of potential pregabalin and duloxetine DDIs on 6-month post-initiation costs. Among

Table 7 Model-based* 6-month post-index all-cause total healthcare costs by index medication and DDI status and difference-in-difference comparison between pregabalin and duloxetine

\begin{tabular}{lll}
\hline & \multicolumn{2}{c}{ All-cause healthcare costs } \\
\cline { 2 - 3 } & Mean & $\begin{array}{l}\text { 95\% confidence } \\
\text { interval }\end{array}$ \\
\hline Pregabalin & $\$ 31,878$ & $\$ 9,545-\$ 106,461$ \\
No DDI present & $\$ 33,942$ & $\$ 8,995-\$ 128,076$ \\
DDI present & $\$ 2,064$ & - \\
Difference** & 0.8159 & - \\
$P$ value & & \\
Duloxetine & $\$ 36,424$ & $\$ 10,847-\$ 122,314$ \\
No DDI present & $\$ 39,445$ & $\$ 11,904-\$ 130,702$ \\
DDI present & $\$ 3,021$ & - \\
Difference** & 0.4208 & - \\
$P$ value & $\$ 2,064$ vs. \\
Difference-in-difference & $\$ 3,021$ & \\
comparison & $p=0.8950$ \\
Statistical differencet & \\
\hline
\end{tabular}

*GLMM model with gamma distribution and log link. Model-based means, standard errors, and confidence intervals were derived from model and transformed into dollar values through exponentiation. All $p$ values are calculated using Wald chi square tests through LSMEANS option. Presence of potential DDI, Drug used (duloxetine versus pregabalin), DDI*Drug interaction term, baseline demographic characteristics, baseline comorbidities present, and pre-index medication utilization controlled for in GLM model.

**Represents the additional healthcare costs associated with the presence of a potential DDI.

$+t$-test, utilizing model estimates, comparing pregabalin difference (DDI minus No DDI) to duloxetine difference (DDI minus No DDI).

DDI, Drug-Drug Interaction.

$\mathrm{Cl}$, Confidence Interval. duloxetine subjects, mean all-cause total healthcare, total medical, inpatient-related, emergency room, and total pharmacy costs were significantly higher for those with a potential DDI than those without. Non-statistically significant cost increases were found between the pregabalin members with and without a potential DDI. These findings are substantiated by the Johnston et al. study that found the combined presence of potential DDIs and potential drug-condition interactions (DCIs) were associated with significantly higher all-cause healthcare costs in duloxetine users but not so in pregabalin users [34].

The non-significant findings of the within-pregabalin cohort comparisons and in the difference-in-difference analysis must be tempered by the small number of pregabalin subjects with a potential DDI $(n=13)$. The increase in costs associated with potential DDIs within the duloxetine cohort, however, cannot be overlooked. Prior studies of chronic pain conditions have found that CYP450 interactions between specific opioids (codeine, fentanyl, hydrocodone, methadone, oxycodone, and tramadol) and concomitant medications that serve as substrates or inhibitors of CYP450 isoenzymes 3A4 and 2D6, including duloxetine, result in higher costs and greater healthcare utilization [50-52].

A retrospective analysis of 170,086 patients using claims data from 2004 to 2008 found significantly higher mean total 6-month costs in chronic pain subjects with an exposure to a potential DDI compared to matched subjects without an exposure (\$8165 vs. $\$ 7498$, respectively; $\mathrm{p}<0.01$ ) [50]. This same study found that potential DDI exposed patients versus non-exposed patients experienced significantly more office visits $(19.10$ vs 18.29; $\mathrm{p}<0.01)$, outpatient visits (6.71 vs 6.39; $\mathrm{p}<0.01)$, emergency department visits (0.46 vs $0.43 ; \mathrm{p}<0.01)$, and inpatient hospitalizations $(0.13$ vs $0.12 ; \mathrm{p}<0.01)$ [50]. The large sample sizes allowed for detection of small differences, making the statistically significant findings difficult to interpret in terms of clinical 
Table 8 Opioid utilization* in pre- and post-index 6-month periods and difference-in-difference comparison between pregabalin and duloxetine

\begin{tabular}{|c|c|c|c|}
\hline & $\begin{array}{l}\text { Long-acting } \\
\text { opioids }\end{array}$ & $\begin{array}{l}\text { Short-acting } \\
\text { less potent } \\
\text { opioids }\end{array}$ & $\begin{array}{l}\text { Short-acting } \\
\text { more potent } \\
\text { opioids }\end{array}$ \\
\hline & $\%$ & $\%$ & $\%$ \\
\hline \multicolumn{4}{|l|}{ Pregabalin } \\
\hline Pre-index & 7.8 & 51.6 & 15.7 \\
\hline Post-index & 10.1 & 53.8 & 17.5 \\
\hline Difference & +2.2 & +2.2 & +1.8 \\
\hline \multicolumn{4}{|l|}{ Duloxetine } \\
\hline Pre-index & 7.0 & 49.6 & 16.6 \\
\hline Post-index & 10.1 & 50.9 & 19.3 \\
\hline Difference & +3.1 & +1.3 & +2.7 \\
\hline $\begin{array}{l}\text { Difference-in-difference } \\
\text { comparison** }\end{array}$ & $\begin{array}{l}2.2 \text { versus } \\
3.1\end{array}$ & 2.2 versus 1.3 & 1.8 versus 2.7 \\
\hline Statistical Difference ${ }^{* *}$ & $\begin{array}{l}t=-1.08 \\
p=0.281\end{array}$ & $\begin{array}{l}t=0.23 \\
p=0.816\end{array}$ & $\begin{array}{l}t=-0.28 \\
p=0.782\end{array}$ \\
\hline
\end{tabular}

*Defined as one or more opioid prescription fills during period.

**Difference in Difference t-statistic derived from a generalized linear mixed model with a logit link and binary distribution adjusting for baseline demographics, Deyo-Charlson comorbidity index, and pre- and post-index non-opioid pain medication utilization

relevance. The increases in healthcare utilization in the presence of potential DDI exposure is directionally consistent with the duloxetine cohort in the present study with regard to percent of patients with inpatient hospitalization and ER visits and mean numbers of visits per member for inpatient hospitalizations, ER visits, and non-physical therapy outpatient visits.

Table 9 Morphine equivalents utilization in pre- and post-index 6-month periods and difference-in-difference comparison between pregabalin and duloxetine

\begin{tabular}{lll}
\hline & \multicolumn{2}{l}{$\begin{array}{l}\text { Morphine equivalents } \\
\text { milligrams/30 days }\end{array}$} \\
\cline { 2 - 3 } & Mean & $\begin{array}{l}\text { Standard } \\
\text { deviation }\end{array}$ \\
\hline Pregabalin & 525.7 & $1,682.0$ \\
Pre-index & 585.9 & $1,539.8$ \\
Post-index & 60.2 & 899.2 \\
Difference & & \\
Duloxetine & 442.6 & $1,208.0$ \\
Pre-index & 619.5 & $1,652.7$ \\
Post-index & 176.9 & 936.5 \\
Difference & 60.2 vs. 176.9 \\
Difference-in-difference comparison* & \\
Statistical difference & $\mathrm{t}=-1.90, \mathrm{p}=0.058$ & \\
\hline
\end{tabular}

*Difference in Difference t-statistic derived from a generalized linear mixed model with an identity link and a Gaussian distribution adjusting for baseline demographics, Deyo-Charlson comorbidity index, and pre- and post-index non-opioid pain medication utilization.
Changes in opioid utilization after initiation of pregabalin or duloxetine, regardless of potential DDI occurrence, were also assessed in the present study. No changes in pre- to post-initiation of long-acting opioid, short-acting opioid, strong opioid, weak opioid, or "any" opioid use were found for pregabalin or duloxetine, as defined by the percentage of patients with one or more pharmacy claims, in the lone head-to-head retrospective analysis of these drugs in a pDPN population [29]. However, when defined as the number of prescriptions filled by opioid users, both pregabalin and duloxetine patients experienced statistically significant increases in mean number of prescriptions filled for short-acting, weak, and "any" opioids [29]. Duloxetine patients also exhibited significantly greater post-index use of long-acting opioids [29]. The difference between the increases observed for pregabalin and duloxetine were not compared. As in the aforementioned study [29], the present study found no difference between pregabalin and duloxetine patients in their respective pre-versus-post differences in the percentage of members with $\geq 1$ pharmacy claims for long-acting opioids, short-acting more potent opioids, or short-acting less potent opioids.

Given that opioids within the same category, such as "long-acting," vary in terms of potency, the present study expanded on the aforementioned research [29] by assessing opioid use as a function of morphine equivalents. A robust difference-in-difference analysis of 6-month preindex and 6-month post-index morphine equivalents used identified a trend toward greater mean morphine equivalents per 30-day post-index increase in use in the duloxetine cohort relative to the pregabalin cohort (176.9 $\mathrm{mg}$ vs. $60.2 \mathrm{mg}$, respectively; $\mathrm{p}=0.058$ ). As opioids vary not only in duration of action but also in potency, this finding, while not providing clarity on the opioid-sparing differences of pregabalin compared to duloxetine, does support the use of morphine equivalents as a preferred method of comparing changes in opioid utilization.

Several limitations must be noted for studies involving administrative claims data. Causal effect cannot be assigned given the observational nature of this study. The use of secondary data is limited by the threat of validity posed by inaccurate data within healthcare information technology systems as well as by differences in coding and documentation practices at the provider, region, or site-specific level. Actual patient behaviors regarding medication consumption cannot be assessed through paid pharmacy claims. Further, reliance on paid pharmacy claims for medication utilization does not capture the use of over-the-counter medications or remedies impactful to the disease state in question. Indirect costs were not considered as part of this study. A reduction in confounding was attempted through the use of 
propensity score matching and multivariate modeling. These approaches are limited by only the covariates included in the statistical models. Even with this attempt at bias reduction, residual confounding from included covariates can still occur and confounding from unknown or unmeasured covariates remains a possibility. The use of "potential" DDIs does not imply the actual occurrence of clinically-impactful DDIs, and therefore limits the interpretation and applicability of the findings. Lastly, given the focus on pDPN patients, the results of this study should not be considered generalizable to other FDA-approved indications or off-label uses.

\section{Conclusion}

The results of this retrospective analysis suggest that the real-world prevalence of potential duloxetine DDIs in a MAPD pDPN population is substantially greater than that of potential pregabalin DDIs. While it is desirable to find infrequent DDIs, the low prevalence of potential pregabalin DDIs places limits on both the performance and sensitivity of within or between drug comparisons. From a clinical perspective, the importance of considering DDIs during treatment selection should be considered due to the significantly higher prevalence of potential DDIs found in pDPN duloxetine users.

\section{Abbreviations}

DDI: Drug-drug interaction; Cl: Confidence interval; CPT: Current Procedural Terminology; DCl: Drug-condition interaction; DE: Dual eligibility; DID: Differencein-difference; ER: Emergency room; FM: Fibromyalgia; GLMM: Generalized linear mixed model; HCU: Healthcare utilization; ICD-9-CM: International Classification of Disease, 9th revision, Clinical Modification; LIS: Low income subsidy; LS: Least square; MAPD: Medicare Advantage Prescription Drug; MEq: Morphine equivalents; mg: Milligrams; pDPN: Painful diabetic peripheral neuropathy; PHI: Protected health information; PS: Propensity score; PT: Physical therapy; SD: Statndard deviation; SNRI: Serotoninnorepinephrine reuptake inhibitors; US: United States.

\section{Competing interests}

This research was conceived, funded, and carried out collaboratively by Humana Inc., Pfizer Inc., and Comprehensive Health Insights Inc. The research concept was approved by the Joint Research Governance Committee of the Humana-Pfizer Research Collaboration, comprised of Humana Inc. and Pfizer Inc. employees, and plans to publish results were made known prior to commencing the study. JE, PM, and BS are employees of Comprehensive Health Insights Inc., a wholly owned subsidiary of Humana Inc., which provides consulting and other research services to pharmaceutical, academic, government, and non-government organizations. In this salaried position, they work with a variety of companies and organizations and are precluded from receiving payment or honoraria directly from these organizations for services rendered. BS is a stockholder of Humana Inc. LN is an employee of Humana Inc. Comprehensive Health Insights Inc. received funding support from Pfizer Inc. in connection with conducting this study and for the development of this article. AS, JC, and BP are employees and stockholders of Pfizer Inc. At the time that this research was conducted, LLT was an employee of Comprehensive Health Insights Inc. The authors declare that they have no non-financial competing interests.

\section{Authors' contributions}

JJE, ABS, JCC, LN, BTS and BP have made substantial contributions to conception, design, and interpretation of data. LLT and PM have made significant contributions to acquisition of data, analysis, and interpretation of data. BTS, PM, and JJE have been involved in drafting the manuscript. All authors have been involved in revising the manuscript critically for important intellectual content. All authors have given final approval of the version to be published and agree to be accountable for all aspects of the work in ensuring that questions related to the accuracy or integrity of any part of the work are appropriately investigated and resolved. All authors read and approved the final manuscript.

\section{Acknowledgements}

The authors wish to thank Mary Costantino, PhD at Comprehensive Health Insights Inc. for medical writing and editorial assistance of this manuscript. MC was funded as a paid employee of Comprehensive Health Insights Inc.

\section{Author details}

${ }^{1}$ Comprehensive Health Insights Inc., 325 West Main Street WFP6W, Louisville, KY 40202, USA. ${ }^{2}$ Pfizer Inc., 235 East 42nd Street, NewYork, NY 10017, USA. ${ }^{3}$ Formerly of Comprehensive Health Insights Inc., 325 West Main Street WFP6W, Louisville, KY 40202, USA. ${ }^{4}$ Humana Inc., 323 West Main Street WFP-05C, Louisville, KY 40202, USA.

Received: 18 May 2014 Accepted: 30 March 2015

Published online: 15 April 2015

\section{References}

1. Thomas PK. Classification, differential diagnosis, and staging of diabetic peripheral neuropathy. Diabetes. 1997:46:S54-7.

2. Freeman R. Autonomic peripheral neuropathy. Lancet. 2005;365(9466):1259-70.

3. Schmader KE. Epidemiology and impact on quality of life of postherpetic neuralgia and painful diabetic neuropathy. Clin J Pain. 2002;18(6):350-4.

4. Boulton AJM, Malik RA, Arezzo JC, Sosenko JM. Diabetic somatic neuropathies. Diabetes Care. 2004;27(6):1458-86.

5. Boulton AJ, Vinik Al, Arezzo JC, Bril V, Feldman EL, Freeman R, et al. Diabetic neuropathies: a statement by the American Diabetes Association. Diabetes Care. 2005;28(4):956-62.

6. Barrett AM, Lucero MA, Le T, Robinson RL, Dworkin RH, Chappell AS. Epidemiology, public health burden, and treatment of diabetic peripheral neuropathic pain: a review. Pain Med. 2007;8 Suppl 2:S50-62.

7. Fischer TZ, Waxman SG. Neuropathic pain in diabetes-evidence for a central mechanism. Nat Rev Neurol. 2010;6(8):462-6.

8. Eaton SE, Harris ND, Rajbhandari SM, Greenwood P, Wilkinson ID, Ward JD, et al. Spinal-cord involvement in diabetic peripheral neuropathy. Lancet. 2001:358(9275):35-6.

9. Hovaguimian $\mathrm{A}$, Gibbons $\mathrm{CH}$. Clinical approach to the treatment of painful diabetic neuropathy. Ther Adv Endocrinol Metab. 2011;2(1):27-38.

10. Vinik Al, Casellini CM. Guidelines in the management of diabetic nerve pain: clinical utility of pregabalin. Diabetes Metab Syndr Obes. 2013;6:57-78.

11. Tesfaye $S$. Advances in the management of diabetic peripheral neuropathy. Curr Opin Support Palliat Care. 2009;3(2):136-43.

12. Gore M, Brandenburg NA, Hoffman DL, Tai K-S, Stacey B. Burden of illness in painful diabetic peripheral neuropathy: the patients' perspectives. J Pain. 2006; (12):892-900.

13. Lesser H, Sharma U, LaMoreaux L, Poole RM. Pregabalin relieves symptoms of painful diabetic neuropathy: a randomized controlled trial. Neurology. 2004;63(11):2104-10.

14. Rosenstock J, Tuchman M, LaMoreaux L, Sharma U. Pregabalin for the treatment of painful diabetic peripheral neuropathy: a double-blind, placebo-controlled trial. Pain. 2004;110(3):628-38.

15. Richter RW, Portenoy R, Sharma U, Lamoreaux L, Bockbrader H, Knapp LE. Relief of painful diabetic peripheral neuropathy with pregabalin: a randomized, placebo-controlled trial. J Pain. 2005;6(4):253-60.

16. Goldstein DJ, Lu Y, Detke MJ, Lee TC, lyengar S. Duloxetine vs. placebo in patients with painful diabetic neuropathy. Pain. 2005;116(1-2):109-18.

17. Raskin J, Pritchett YL, Wang F, D'Souza DN, Waninger AL, lyengar S, et al. A double-blind, randomized multicenter trial comparing duloxetine with placebo in the management of diabetic peripheral neuropathic pain. Pain Med. 2005;6(5):346-56.

18. Wernicke JF, Pritchett YL, D'Souza DN, Waninger A, Tran P, lyengar S, et al. A randomized controlled trial of duloxetine in diabetic peripheral neuropathic pain. Neurology. 2006;67(8):1411-20.

19. Hoy SM. Tapentadol extended release: in adults with chronic pain. Drugs. 2012;72(3):375-93. 
20. Schwartz S, Etropolski M, Shapiro DY, Okamoto A, Lange R, Haeussler J, et al. Safety and efficacy of tapentadol ER in patients with painful diabetic peripheral neuropathy: results of a randomized-withdrawal, placebo-controlled trial. Curr Med Res Opin. 2011;27(1):151-62.

21. Jensen TS, Backonja M-M, Hernández Jiménez S, Tesfaye S, Valensi P, Ziegler D. New perspectives on the management of diabetic peripheral neuropathic pain. Diabetes Vasc Dis Res. 2006;3(2):108-19.

22. Zin CS, Nissen LM, O'Callaghan JP, Duffull SB, Smith MT, Moore BJ. A randomized, controlled trial of oxycodone versus placebo in patients with postherpetic neuralgia and painful diabetic neuropathy treated with pregabalin. J Pain. 2010;11(5):462-71.

23. Gimbel JS, Richards P, Portenoy RK. Controlled-release oxycodone for pain in diabetic neuropathy: a randomized controlled trial. Neurology. 2003:60(6):927-34.

24. Watson CPN, Moulin D, Watt-Watson J, Gordon A, Eisenhoffer J. Controlledrelease oxycodone relieves neuropathic pain: a randomized controlled trial in painful diabetic neuropathy. Pain. 2003;105(1-2):71-8.

25. Roy S, Loh HH. Effects of opioids on the immune system. Neurochem Res. 1996;21(11):1375-86

26. Lee C, Ludwig S, Duerksen DR. Low serum cortisol associated with opioid use: case report and review of the literature. Endocrinol. 2002;12:5-8.

27. Daniell HW. Opioid endocrinopathy in women consuming prescribed sustained-action opioids for control of nonmalignant pain. J Pain. 2008;9(1):28-36.

28. Pergolizzi J, Böger RH, Budd K, Dahan A, Erdine S, Hans G, et al. Opioids and the management of chronic severe pain in the elderly: consensus statement of an International Expert Panel with focus on the six clinically most often used World Health Organization Step III opioids (buprenorphine, fentanyl, hydromorphone, methadone, morphine, oxycodone). Pain Pract. 2008;8(4):287-313.

29. Gore M, Zlateva G, Tai KS, Chandran AB, Leslie D. Retrospective evaluation of clinical characteristics, pharmacotherapy and healthcare resource use among patients prescribed pregabalin or duloxetine for diabetic peripheral neuropathy in usual care. Pain Pract. 2011;11(2):167-79.

30. Gore M, Tai KS, Chandran A, Zlateva G, Leslie D. Clinical comorbidities, treatment patterns, and healthcare costs among patients with fibromyalgia newly prescribed pregabalin or duloxetine in usual care. J Med Econ. 2012:15(1):19-31.

31. Gore M, Sadosky A, Tai K-S, Stacey B. A retrospective evaluation of the use of gabapentin and pregabalin in patients with postherpetic neuralgia in usual-care settings. Clin Ther. 2007;29:1655-70.

32. Gore M, Tai K-S, Zlateva G, Chandran AB, Leslie D. Clinical characteristics, pharmacotherapy, and healthcare resource use among patients with diabetic neuropathy newly prescribed pregabalin or gabapentin. Pain Pract. 2011;11:1-12.

33. Wu N, Chen SY, Hallett LA, Boulanger L, Fraser KA, Patel CK, et al. Opioid utilization and health-care costs among patients with diabetic periphera neuropathic pain treated with duloxetine vs. other therapies. Pain Pract. 2011;11:48-56

34. Johnston SS, Udall M, Cappelleri JC, Johnson BH, Shrady G, Chu BC, et al. Cost comparison of drug-drug and drug-condition interactions in patients with painful diabetic peripheral neuropathy treated with pregabalin versus duloxetine. Am J Health Syst Pharm. 2013;70(24):2207-17.

35. Dworkin RH, Panarites CJ, Armstrong EP, Malone DC, Pham SV. Healthcare utilization in people with postherpetic neuralgia and painful diabetic peripheral neuropathy. J Am Geriatr Soc. 2011;59(5):827-36.

36. Burke JP, Sanchez RJ, Joshi AV, Cappelleri JC, Kulakodlu M, Halpern R. Health care costs in patients with painful diabetic peripheral neuropathy prescribed pregabalin or duloxetine. Pain Pract. 2012;12(3):209-18.

37. Deyo RA, Cherkin DC, Ciol MA. Adapting a clinical comorbidity index for use with ICD-9-CM administrative databases. J Clin Epidemiol. 1992;45:613-9.

38. Klabunde CN, Potosky AL, Legler JM, Warren JL. Development of a comorbidity index using physician claims data. J Clin Epidemiol. 2000;53:1258-67.

39. Von Korff MV, Saunders K, Thomas Ray G, Boudreau D, Campbell C, Merrill J, et al. De facto long-term opioid therapy for non-cancer pain. Clin J Pain. 2008;24(6):521-7.

40. Rosenbaum PR, Rubin DB. The central role of the propensity score in observational studies for causal effects. Biometrika. 1983;70:41-55.
41. D'Agostino Jr RB. Propensity score methods for bias reduction in the comparison of a treatment to a non-randomized control group. Stat Med. 1998;17:2265-81.

42. Rosner B. Fundamentals of Biostatistics. 7th ed. Boston, MA: Brooks/Cole Cengage Learning; 2010.

43. Jones AM, Rice N, Bago d'Uva T, Balia S. Applied Health Economics. 2nd ed. New York, NY: Routledge; 2013.

44. Lyrica ${ }^{\circledast}$ (pregabalin) package insert. New York, IN: Pfizer Inc.; 2013

45. Cymbalta $^{\oplus}$ (duloxetine) package insert. Indianapolis, IN: Eli Lilly and Company; 2012.

46. Pergolizzi Jr JV, Labhsetwar SA, Puenpatom RA, Joo S, Ben-Joseph RH, Summers KH. Prevalence of exposure to potential CYP450 pharmacokinetic drug-drug interactions among patients with chronic low back pain taking opioids. Pain Pract. 2011;11(3):230-9.

47. Pergolizzi Jr JV, Labhsetwar SA, Puenpatom RA, Joo S, Ben-Joseph R, Summers KH. Exposure to potential CYP450 pharmacokinetic drug-drug interactions among osteoarthritis patients: incremental risk of multiple prescriptions. Pain Pract. 2011;11(4):325-36.

48. Mallet $L$, Spinewine A, Huang A. The challenge of managing drug interactions in elderly people. Lancet. 2007;370(9582):185-91.

49. Hines LE, Murphy JE. Potentially harmful drug-drug interactions in the elderly: a review. Am J Geriatr Pharmacother. 2011;9(6):364-77.

50. Summers KH, Puenpatom RA, Rajan N, Ben-Joseph R, Ohsfeldt R. Economic impact of potential drug-drug interactions in opioid analgesics. J Med Econ. 2011;14:390-6.

51. Pergolizzi Jr JV, Labhsetwar SA, Puenpatom RA, Ben-Joseph R, Ohsfeldt R, Summers $\mathrm{KH}$. Economic impact of potential CYP450 pharmacokinetic drug-drug interactions among chronic low back pain patients taking opioids. Pain Pract. 2012;12:45-56.

52. Pergolizzi Jr JV, Labhsetwar SA, Puenpatom RA, Ben-Joseph R, Ohsfeldt R, Summers $\mathrm{KH}$. Economic impact of potential drug-drug interactions among osteoarthritis patients taking opioids. Pain Pract. 2012;12:33-44.

\section{Submit your next manuscript to BioMed Central and take full advantage of:}

- Convenient online submission

- Thorough peer review

- No space constraints or color figure charges

- Immediate publication on acceptance

- Inclusion in PubMed, CAS, Scopus and Google Scholar

- Research which is freely available for redistribution 